\title{
Genetic determinants of serum vitamin B12 and their relation to body mass index
}

\author{
Kristine H. Allin ${ }^{1} \cdot$ Nele Friedrich ${ }^{2,3} \cdot$ Maik $^{\text {Pietzner }}{ }^{2} \cdot$ Niels Grarup $^{1}$ • \\ Betina H. Thuesen ${ }^{3}$ - Allan Linneberg ${ }^{3,4}$ - Charlotta Pisinger $^{3}$ - Torben Hansen ${ }^{1,5}$. \\ Oluf Pedersen $^{1,6}$ - Camilla H. Sandholt ${ }^{1}$
}

Received: 6 April 2016/Accepted: 28 November 2016/Published online: 19 December 2016

(c) The Author(s) 2016. This article is published with open access at Springerlink.com

\begin{abstract}
Lower serum vitamin B12 levels have been related to adverse metabolic health profiles, including adiposity. We used a Mendelian randomization design to test whether this relation might be causal. We included two Danish population-based studies $\left(n_{\text {total }}=9311\right)$. Linear regression was used to test for associations between (1) serum vitamin B12 levels and body mass index (BMI), (2) genetic variants and serum vitamin B12 levels, and (3) genetic variants and BMI. The effect of a genetically determined decrease in serum vitamin $\mathrm{B} 12$ on BMI was estimated by instrumental variable regression. Decreased serum vitamin B12 associated with increased BMI $\left(P<1 \times 10^{-4}\right)$. A genetic risk score based on eight vitamin $\mathrm{B} 12$ associated variants associated strongly with serum vitamin B12 $\left(P<2 \times 10^{-43}\right)$, but not with BMI
\end{abstract}

Electronic supplementary material The online version of this article (doi:10.1007/s10654-016-0215-x) contains supplementary material, which is available to authorized users.

Kristine H. Allin

kristine.allin@sund.ku.dk

1 The Novo Nordisk Foundation Center for Basic Metabolic Research, Section of Metabolic Genetics, Faculty of Health and Medical Sciences, University of Copenhagen,

Universitetsparken 1, 2100 Copenhagen, Denmark

2 Institute of Clinical Chemistry and Laboratory Medicine, University Medicine Greifswald, Greifswald, Germany

3 Research Centre for Prevention and Health, The Capital Region of Denmark, Copenhagen, Denmark

4 Department of Clinical Experimental Research, Rigshospitalet, Glostrup, Denmark

5 Faculty of Health Sciences, University of Southern Denmark, Odense, Denmark

6 Faculty of Health Sciences, University of Aarhus, Aarhus, Denmark
$(P=0.91)$. Instrumental variable regression showed that a $20 \%$ decrease in serum vitamin B12 was associated with a $0.09 \mathrm{~kg} / \mathrm{m}^{2} \quad(95 \%$ CI $0.05 ; 0.13)$ increase in BMI $\left(P=3 \times 10^{-5}\right)$, whereas a genetically induced $20 \%$ decrease in serum vitamin B12 had no effect on BMI [ -0.03 (95\% CI $\left.-0.22 ; 0.16) \mathrm{kg} / \mathrm{m}^{2}\right](\mathrm{P}=0.74)$. Nevertheless, the strongest serum vitamin B12 variant, FUT2 rs602662, which was excluded from the $\mathrm{B} 12$ genetic risk score due to potential pleiotropic effects, showed a per allele effect of $0.15 \mathrm{~kg} / \mathrm{m}^{2}$ (95\% CI $0.01 ; 0.32)$ on BMI $(P=0.03)$. This association was accentuated including two German cohorts $\left(\mathrm{n}_{\text {total }}=5050\right)$, with a combined effect of $0.19 \mathrm{~kg} / \mathrm{m}^{2}$ (95\% CI 0.08 ; 0.30) $\left(P=4 \times 10^{-4}\right)$. We found no support for a causal role of decreased serum vitamin B12 levels in obesity. However, our study suggests that FUT2, through its regulation of the crosstalk between gut microbes and the human host, might explain a part of the observational association between serum vitamin B12 and BMI.

Keywords BMI - Cobalamin $\cdot$ FUT2 $\cdot$ Mendelian randomization $\cdot$ Secretor/nonsecretor $\cdot$ Vitamin B12

\section{Introduction}

Human obesity is a highly heterogeneous disorder where multiple factors, especially related to lifestyle, contribute to the pathogenic development. A high degree of heritability has also been established [1], and a substantial number of genetic variants associated with obesity-related phenotypes have been identified $[2,3]$. The pathways and mechanisms underlying these genetic associations have not been fully elucidated, but central nervous system and adipose tissue biology have been highlighted. Nevertheless, the genetic variants only explain a small fraction of the phenotypic 
variance [2, 3], indicating that yet undiscovered mechanisms could be essential in the obesity pathogenesis.

Vitamin B12 (cobalamin) is solely produced by Bacteria and Archaea [4] and the only natural source of this vitamin for humans are foods of animal origin such as meat, fish, eggs, and dairy products. In humans, vitamin B12 functions as an essential coenzyme in various cellular functions such as cellular energy metabolism, DNA synthesis and methylation, as well as protein, lipid, and carbohydrate synthesis [5]. Several studies have observed associations between lower circulating vitamin B12 levels and adverse metabolic health profiles, with insulin resistance, cardiovascular disorders, and adiposity as important features [6-10].

The heritability of serum vitamin B12 levels has in a study of mono- and dizygotic twins been estimated to be $59 \%$ [11]. Several studies have, in genome-wide association studies (GWAS) and using sequencing efforts, identified at least 12 genetic variants robustly associated with circulating vitamin B12 levels [12-16]. Interestingly, despite using hypothesis-free genome-wide approaches, the vast majority of the identified single nucleotide polymorphisms (SNP) are situated in or near genes encoding proteins responsible for the absorption, processing, and coenzymatic activity of vitamin B12 [16].

These genetic variants allow the construction of a genetic risk score (GRS) that can serve as a relatively unbiased instrument for serum vitamin B12 levels in a Mendelian randomization study [17]. Here, we used this instrument in two Danish population-based cohorts conducted at a time point where legislation did not allow fortification with vitamin B12 (or folate) of food items [18], to test the hypothesis that lower serum vitamin B12 levels are causally related to obesity.

\section{Materials and methods}

\section{Study populations}

The Danish population-based Inter99 study (ClinicalTrials.gov ID-no: NCT00289237) is a non-pharmacological intervention study for ischemic heart disease, initiated in 1999 at the Research Centre for Prevention and Health, Glostrup, Denmark. A random sample of 13,016 individuals living in Copenhagen County from seven different age groups (30-60 years, grouped with 5 year intervals) was drawn from the Civil Registration System and 6784 of these attended the health examination [19].

The Danish Health2006 cohort is a population-based study of general health and chronic diseases such as type 2 diabetes and cardiovascular disease in individuals aged 18-69 years. The study was initiated in 2006 at Research Centre for Prevention and Health, Glostrup, Denmark, and participants were drawn as a random sample of the background population living in the south-western part of the greater Copenhagen area. A total of 7770 were invited to participate and 3471 entered and participated in the health examination [20].

The Study of Health in Pomerania (SHIP-0) is a population-based study in West Pomerania, a rural region in north-east Germany [21], where 6265 individuals, aged 20-79 years, were invited, of which 4308 participated. Data collection was performed between 1997 and 2001. For SHIP-TREND, an independent random sample of 8826 individuals aged 20-79 years was drawn from the same region between 2008 and 2012, and 4420 participated.

All participants gave written informed consent. The studies were approved by local ethics committees and conformed to the principles of the declaration of Helsinki.

\section{Anthropometric, biochemical, and lifestyle measures}

In all cohorts, body weight $(\mathrm{kg})$, waist circumference $(\mathrm{cm})$, hip circumference $(\mathrm{cm})$ and height $(\mathrm{cm})$ were measured in light indoor clothes and without shoes. Body mass index (BMI) was calculated as body weight divided by the squared height in meters $\left(\mathrm{kg} / \mathrm{m}^{2}\right)$. In the Health2006 cohort body fat percentage was measured using bioimpedance.

For the Danish cohorts, vitamin B12 was measured in serum obtained from fasting blood samples, stored at $-20{ }^{\circ} \mathrm{C}$ until the analyses were performed in 2008 . For the German cohorts, blood samples were analyzed immediately or stored at $-80^{\circ}$ until analysis. In all cohorts, serum vitamin B12 was measured using a competitive chemiluminescent enzyme immunoassay (CENTAUR or Dimension Vista platform, Siemens Healthcare Diagnostics), performed by Institute of Clinical Chemistry and Laboratory Medicine, University Medicine Greifswald, Germany.

In the Danish cohorts, all lifestyle factors were estimated from self-reported questionnaire data. A three-point dietary score was developed based on a 48-item food frequency questionnaire and categorized as; (1) unhealthy, (2) moderately healthy, and (3) healthy [22]. Alcohol intake was estimated as the total units of alcohol (1 unit equals 12 grams of alcohol) per week. The level of physical activity was estimated summing the time spent actively commuting (min/week) and time spent on leisure time physical activity (min/week). Subsequently, four categories were created; (1) 0-2 h/week, (2) 2-4 h/week, (3) 4-7 h/week, and (4) 7-12 h/week [23]. Smoking habits were divided into three classes: (1) never smoked, (2) former smoker, (3) daily and occasional smoker [24].

\section{Genotyping, SNP selection, and genetic risk score}

Genotyping is described in detail in Supplemental Materials and Methods. Inter99 and Health2006 were genotyped 
using the Human Exome BeadChip on an Illumina HiScan system (Illumina). In Inter99 a total of 6161 individuals were genotyped and passed all quality control criteria and the corresponding number was 2914 for Health2006. The average call rate for all SNPs on the Human Exome BeadChip was $99.0 \%$.

The SNPs for the GRS were selected based on a joint Icelandic and Danish sequencing initiative reporting 11 loci associated genome-wide significantly $\left(P<5 \times 10^{-8}\right)$ with serum vitamin B12 levels [16], of which six were validation of previous GWAS in European and Chinese populations [12-15]. Of the 11 serum vitamin B12 associated SNPs [16], ten (MMAA rs2270655, MUT rs1141321, CUBN rs1801222, TCN1 rs34324219, CLYBL rs41281112, ABCD4 rs3742801, CD320 rs2336573, TCN2 rs1131603, FUT6 rs778805 and FUT2 rs602662) could be retrieved from the Human Exome BeadChip, whereas one (MMACHC rs12272669) was not represented. The SNP quality of the ten SNPs retrieved from the Human Exome BeadChip was estimated based on genotype call rate $(>95 \%)$, Hardy-Weinberg equilibrium (HWE) $(P>0.005)$, or cross-hybridization with the $\mathrm{X}$-chromosome, and all ten SNPs passed these filters (HWE; $P>0.04$, call rate $>98.1 \%$ ).

The genotypes were coded according to number of serum vitamin B12 decreasing alleles. For four SNPs the minor allele, and for six SNPs the major allele, were considered the effect allele which had frequencies ranging from 3.0 to $96.9 \%$ in Inter99 and from 3.0 to $97.2 \%$ in Health2006. A full vitamin B12 GRS (full-B12 GRS) was calculated by summarizing the number of serum vitamin B12 decreasing alleles for the ten SNPs for each individual. Additionally, a B12 GRS without FUT2 rs602662 and FUT6 rs778805 (B12 GRS) was constructed to circumvent the pleiotropic effects of FUT2 and FUT6.

SHIP-0 was genotyped using the Affymetrix GenomeWide Human SNP Array 6.0. FUT2 rs602662 was imputed based on the $1000 \mathrm{G}$ project (phase 1, European superpopulation, March 2012 release [25]) with an imputation quality score of 0.99 . Genotype data were available for 4064 subjects. Only a subset of SHIP-TREND $(\mathrm{n}=986)$ was genotyped. This was done using the Illumina Human Omni 2.5 array, and rs602662 was directly genotyped with a callrate of $99.8 \%$ obeying HWE $(\mathrm{P}=0.02)$.

\section{Statistical analyses}

The statistical analyses were performed using the statistical software R version 3.1.1 (http://www.r-project.org/) and Stata (version 13.1; StataCorp). $P$ values below 0.05 were considered statistically significant. Linear regression models (adjusted for age and sex) were used to test for association between (1) serum vitamin B12 levels and
BMI, (2) SNPs or B12 GRS and serum vitamin B12 levels, and (3) SNPs or B12 GRS and BMI. The serum vitamin B12 and BMI association was additionally tested using multifactor adjustment (diet, alcohol consumption, physical activity, and smoking). Association between the B12 GRS and potential confounders were tested using linear regression models for continuous outcomes (age and alcohol consumption) and logistic regression for categorical outcomes (diet, physical activity, and smoking). Associations between individual SNPs or the B12 GRS and serum vitamin B12 levels/BMI were analyzed using an additive model. The FUT2 rs602662 genotype was additionally analyzed using a dominant model. Explained phenotypic variance $\left(\mathrm{R}^{2}\right)$ was estimated using unadjusted linear models. The effect of a genetically determined decrease in serum vitamin B12 on BMI was estimated using unadjusted instrumental variable regressions. These were performed using the ivreg function of the 'AER' package in $\mathrm{R}$ and the ivregress command in Stata, both using two-stage least-squares regression. The strengths of the instruments were evaluated by F-statistics from first stage regressions. In addition to using the B12 GRS as an instrument, we also used each of the variants as separate instruments, which is equivalent to using a GRS where each variant is weighted based on its association with serum vitamin B12 [26]. To test for directional pleiotropy and to evaluate the robustness of our findings, we also applied MR-Egger regression. Pleiotropy was evaluated by the MR-Egger test which tests whether the intercept differs from zero and by visually inspecting Funnel plots for asymmetry [26]. The combined effect across study populations was estimated using fixed-effect model meta-analyses performed by the rma function of the 'metafor' package in R. Linkage disequilibrium was estimated using SNAP (http://www.broadinstitute.org/mpg/snap/) in data from the European panel of HapMap phase 3 data.

Data on BMI from the Genetic Investigation of Anthropometric Traits (GIANT) consortium was obtained from the European ancestry dataset (http://www.broad institute.org/collaboration/giant/index.php/GIANT_con sortium_data_files).

\section{Results}

The phenotypic characteristics of the two Danish cohorts, Inter99 and Health2006, are shown in Supplementary Table 1. Individuals were middle-aged, with a median BMI of 25.6 and $25.2 \mathrm{~kg} / \mathrm{m}^{2}$, respectively. Serum vitamin B12 levels were higher in Health2006 compared to Inter99, but similar among women and men within cohorts (Supplemental Table 1). 


\section{Observational association between serum vitamin B12 levels and BMI}

Decreasing levels of serum vitamin B12 were associated with increasing BMI in the two Danish cohorts (Fig. 1). A BMI difference of $0.48(95 \%$ CI $0.19 ; 0.76) \mathrm{kg} / \mathrm{m}^{2}$ and $0.64(95 \%$ CI $0.26 ; 1.02) \mathrm{kg} / \mathrm{m}^{2}$ was observed when comparing the first and third tertile of serum vitamin B12 levels in Inter99 and Health2006, respectively. Accordingly, a 20\% decrease in serum vitamin B12 levels was associated with an increase in BMI of 0.10 (95\% CI 0.05; $0.14) \mathrm{kg} / \mathrm{m}^{2}\left(P=1 \times 10^{-4}\right)$ in Inter99 and $0.19(95 \% \mathrm{CI}$ $0.10 ; 0.27) \mathrm{kg} / \mathrm{m}^{2}\left(P=3 \times 10^{-5}\right)$ in Health2006. Linear regression model additionally adjusted for lifestyle factors (diet, alcohol consumption, physical activity, and smoking), as well as exclusion of type 2 diabetes patients receiving anti-diabetic treatment $\left(\mathrm{n}_{\text {Inter } 99}=71\right.$, $\mathrm{n}_{\text {Health2006 }}=66$ ) showed similar results (Supplemental Table 2).

In addition to BMI, decreasing levels of serum vitamin B12 were associated with increasing waist circumference and body fat percentage. A $20 \%$ decrease in serum vitamin B12 levels was associated with an increase in waist circumference of $0.23 \quad(95 \%$ CI $0.11 ; 0.35) \quad \mathrm{cm}$ $\left(P=3 \times 10^{-4}\right)$ in Inter99 and $0.46(95 \%$ CI $0.24 ; 0.69)$ $\mathrm{cm}\left(P=6 \times 10^{-5}\right)$ in Health2006. Body fat percentage was only measured in Health2006, where a $20 \%$ decrease in serum vitamin B12 levels was associated with an increase in body fat percentage of 0.29 (95\% CI 0.16 ; $0.42) \%\left(P=1 \times 10^{-5}\right)$. Decreasing levels of serum vitamin B12 were also associated with increasing waist/hip ratio in Health2006 $(P=0.03)$ but not in inter99 $(P=0.14)$.

\section{Genotypes and serum vitamin B12 levels}

The ten SNPs included in the full-B12 GRS each associated with decreased serum vitamin B12 levels in both Inter99 and Health2006 (Supplemental Table 3). The SNP showing the strongest association was FUT2 rs602662 with a per G-allele decrease in serum vitamin B12 of $-10(95 \%$ $\mathrm{CI}-11 ;-8) \%\left(P=3 \times 10^{-37}\right)$ according to an additive model in Inter99. However, the median serum vitamin B12 levels suggested that a dominant model would be more appropriate. Comparing homozygous/heterozygous G-allele carriers with homozygous A-allele carriers showed a serum vitamin B12 level decrease of -20 (95\% CI -22 ; $-17) \%\left(P=1 \times 10^{-51}\right)$. A similar association pattern was observed in Health2006 (Supplemental Table 3).

The full-B12 GRS associated strongly with serum vitamin B12 levels, with a per effect allele decrease of -7 $(95 \% \mathrm{CI}-8 ;-7) \%\left(P=5 \times 10^{-104}\right)$ in Inter99, and a similar association was observed in Health2006 (Supplemental Table 4). However, the overall aim of constructing a GRS was to obtain an instrument for serum vitamin B12 levels not influenced by potential confounders. FUT2 and FUT6 are known to determine ABH antigen secretor/nonsecretor status and be involved in the creation of Lewis antigens, respectively. Hence, both could have pleiotropic effects, and therefore, we also analyzed a GRS excluding
Fig. 1 Observational association between serum vitamin B12 levels divided in tertiles and BMI. Dots and triangles indicate mean BMI and error bars represent standard errors. Black dots represent Inter99 and grey triangles represent Health2006. $P$ values are from age and sex adjusted linear regression of BMI on serum vitamin B12 levels

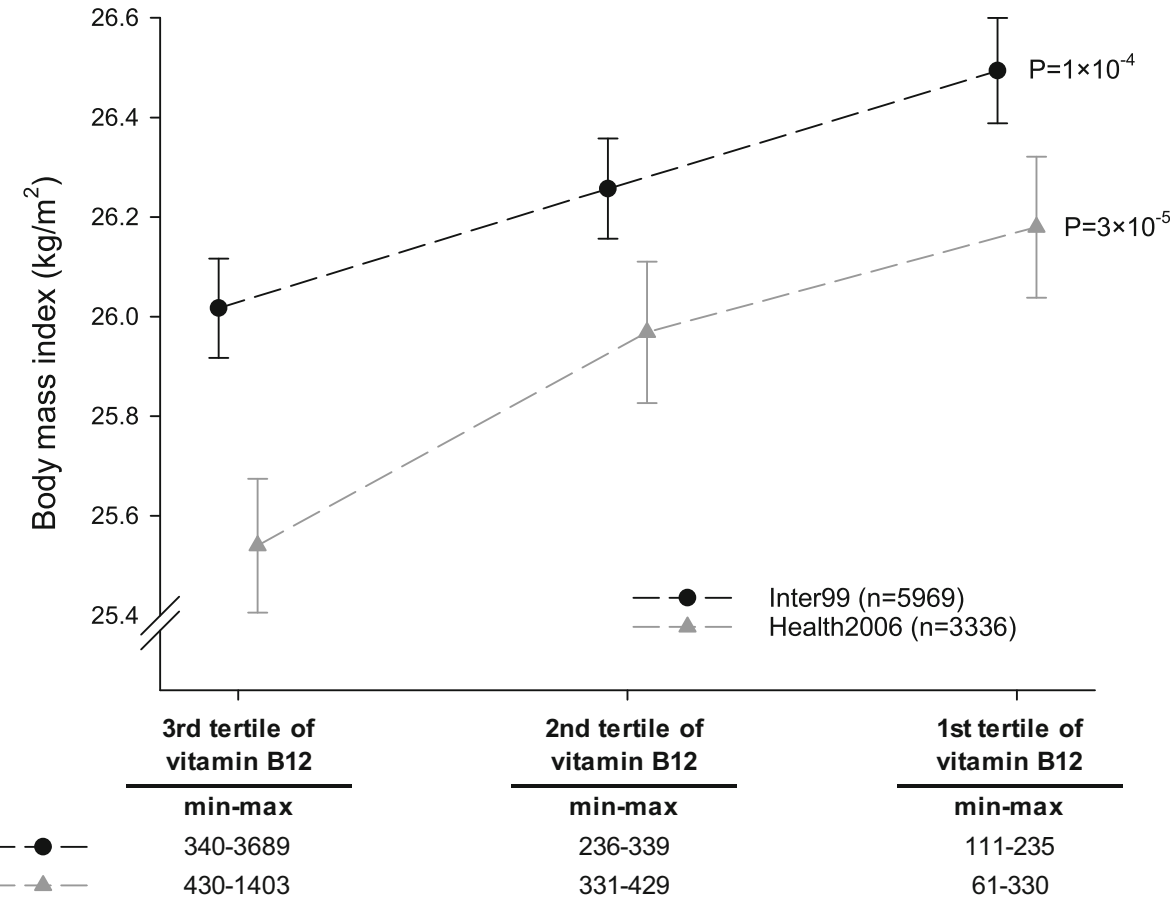


FUT2 rs602662 and FUT6 778805 (B12 GRS). The B12 GRS was not associated with potential confounders including age, sex, diet, alcohol consumption, physical activity, and smoking (Table 1). The proportion of physically inactive individuals was seemingly different among the B12 GRS allele groups, however, the direction of effect was opposite in the two cohorts, which may suggest that this is a chance finding (type 1 error). The B12 GRS showed a per allele decrease in serum vitamin B12 of -7 $(95 \% \mathrm{CI}-8 ;-7) \%\left(P=2 \times 10^{-72}\right)$ in Inter99, and -6 $(95 \%$ CI $-7 ;-5) \%\left(P=2 \times 10^{-43}\right)$ in Health2006 (Fig. 2; Supplemental Table 4). The F-statistics for the models were 331 in Inter99 and 197 in Health2006. The B12 GRS explained 5.5 and $6.7 \%$ of the phenotypic variance in serum vitamin B12 in Inter99 and Health2006, respectively (Supplemental Table 4).

\section{B12 GRS and BMI}

If decreased levels of serum vitamin B12 cause increased BMI, the B12 GRS would, given its strong association with serum vitamin B12 levels, expectedly also associate with increased BMI. However, we observed no association between the B12 GRS and BMI in either Inter99 or Health2006. A fixed-effect model was used to combine the effect sizes from the two cohorts and resulted in a per allele effect of $0.00(95 \%$ CI $-0.07 ; 0.06) \mathrm{kg} / \mathrm{m}^{2}(P=0.91)$ (Table 2). Accordingly, when analyzed separately, none of the eight SNPs included in the B12 GRS associated with $\mathrm{BMI}$ in the two cohorts (data not shown).

\section{Observational versus genetic associations}

To directly compare the observational effect of decreased serum vitamin B12 levels on BMI with the effect of genetically induced low serum vitamin B12 levels on BMI, we performed an instrumental variable analysis. The combined observational effect of a $20 \%$ decrease in serum vitamin $\mathrm{B} 12$ on $\mathrm{BMI}$ in the two cohorts was an increase of $0.09(95 \%$ CI $0.05 ; 0.13) \mathrm{kg} / \mathrm{m}^{2},\left(P=3 \times 10^{-5}\right)$, whereas a genetically induced $20 \%$ decrease in serum vitamin B12 had no effect on BMI $\left(-0.03(95 \%\right.$ CI $-0.22 ; 0.16) \mathrm{kg} / \mathrm{m}^{2}$, $P=0.74)$. Since we observed an association between decreased serum vitamin B12 levels and BMI but no association between genetically induced decreased serum vitamin B12 levels and BMI, the present results do not support a causal role of serum vitamin B12 levels on BMI. However, as the observational estimate (0.09) is included in the $95 \% \mathrm{CI}$ of the genetic estimate $(-0.22 ; 0.16)$, we cannot completely rule out causality (Fig. 3 ).

\section{FUT2 genotype and BMI}

We hypothesized that FUT2 rs602662, through yet undescribed pleiotropic effects, could explain a portion of the observational effect between serum vitamin B12 and BMI given the strong association with serum vitamin B12

Table 1 Association between the B12 GRS and potential confounders

\begin{tabular}{|c|c|c|c|c|c|c|}
\hline \multirow[b]{2}{*}{ Inter99 } & \multicolumn{5}{|c|}{ Number of vitamin B12 decreasing alleles } & \multirow[b]{2}{*}{$P_{\text {trend }}$} \\
\hline & 5 or less $(n=785)$ & $6(\mathrm{n}=1377)$ & $7(\mathrm{n}=1797)$ & $8(\mathrm{n}=1313)$ & 9 or more $(n=809)$ & \\
\hline Women, n (\%) & $420(54)$ & $678(50)$ & $928(52)$ & $647(49)$ & $431(53)$ & 0.39 \\
\hline Age, years & $45(40-50)$ & $45(40-50)$ & $45(40-50)$ & $45(40-55)$ & $45(40-50)$ & 0.75 \\
\hline Unhealthy diet, $\mathrm{n}(\%)^{\mathrm{a}}$ & $117(16)$ & $195(15)$ & $278(16)$ & $210(17)$ & $126(16)$ & 0.52 \\
\hline Alcohol consumption, units per week ${ }^{b}$ & $7(3-14)$ & $7(2-14)$ & $7(2-14)$ & $6(2-14)$ & $6(3-14)$ & 0.93 \\
\hline Physical inactivity, $\mathrm{n}(\%)^{\mathrm{c}}$ & $269(36)$ & $452(35)$ & $598(36)$ & $408(33)$ & $239(32)$ & 0.07 \\
\hline Current smokers, $\mathrm{n}(\%)^{\mathrm{d}}$ & $330(43)$ & $507(37)$ & $706(40)$ & $517(40)$ & $319(40)$ & 0.23 \\
\hline Health2006 & 5 or less $(n=370)$ & $6(\mathrm{n}=630)$ & $7(\mathrm{n}=842)$ & $8(\mathrm{n}=624)$ & 9 or more $(\mathrm{n}=391)$ & $P_{\text {trend }}$ \\
\hline Women, n (\%) & $200(54)$ & $342(54)$ & $446(53)$ & $348(56)$ & $224(57)$ & 0.49 \\
\hline Age, years & $49(41-60)$ & $51(40-61)$ & $51(41-60)$ & $50(41-60)$ & $50(40-60)$ & 0.52 \\
\hline Unhealthy diet, $\mathrm{n}(\%)^{\mathrm{a}}$ & $24(7)$ & $44(7)$ & $53(6)$ & $43(7)$ & $23(6)$ & 0.89 \\
\hline Alcohol consumption, units per week ${ }^{b}$ & $6(2-13)$ & $7(2-14)$ & $6(2-13)$ & $6(2-12)$ & $6(2-14)$ & 0.25 \\
\hline Physical inactivity, $\mathrm{n}(\%)^{\mathrm{c}}$ & $146(40)$ & $275(45)$ & $388(46)$ & $304(49)$ & $189(49)$ & 0.003 \\
\hline Current smokers, $\mathrm{n}(\%)^{\mathrm{d}}$ & $95(26)$ & $167(27)$ & $202(24)$ & $173(28)$ & $98(25)$ & 0.87 \\
\hline
\end{tabular}

Values are median (IQR) unless otherwise specified. $P_{\text {trend }}$ are from linear regressions for continuous potential confounders (age and alcohol) and from logistic regressions for categorical potential confounders: ${ }^{\mathrm{a}}$ Unhealthy diet vs. moderately healthy and healthy diet; ${ }^{\mathrm{b}} 1 \mathrm{unit}=12 \mathrm{~g}$ of alcohol; ${ }^{\mathrm{c}}$ Physical activity $<4 \mathrm{~h}$ per week versus $\geq 4 \mathrm{~h}$ per week; ${ }^{\mathrm{d}}$ Current smokers versus former and never smokers 
Fig. 2 Association between the B12 GRS and serum vitamin B12 levels. Vitamin B12 decreasing alleles ranged from 2 to 13 . Individuals with $\leq 5$ or $\geq 9$ alleles were grouped to obtain a reasonable number of individuals in each group. Dots and triangles indicate median serum vitamin B12 and error bars indicate interquartile range. $P$ values are from age and sex adjusted linear regression

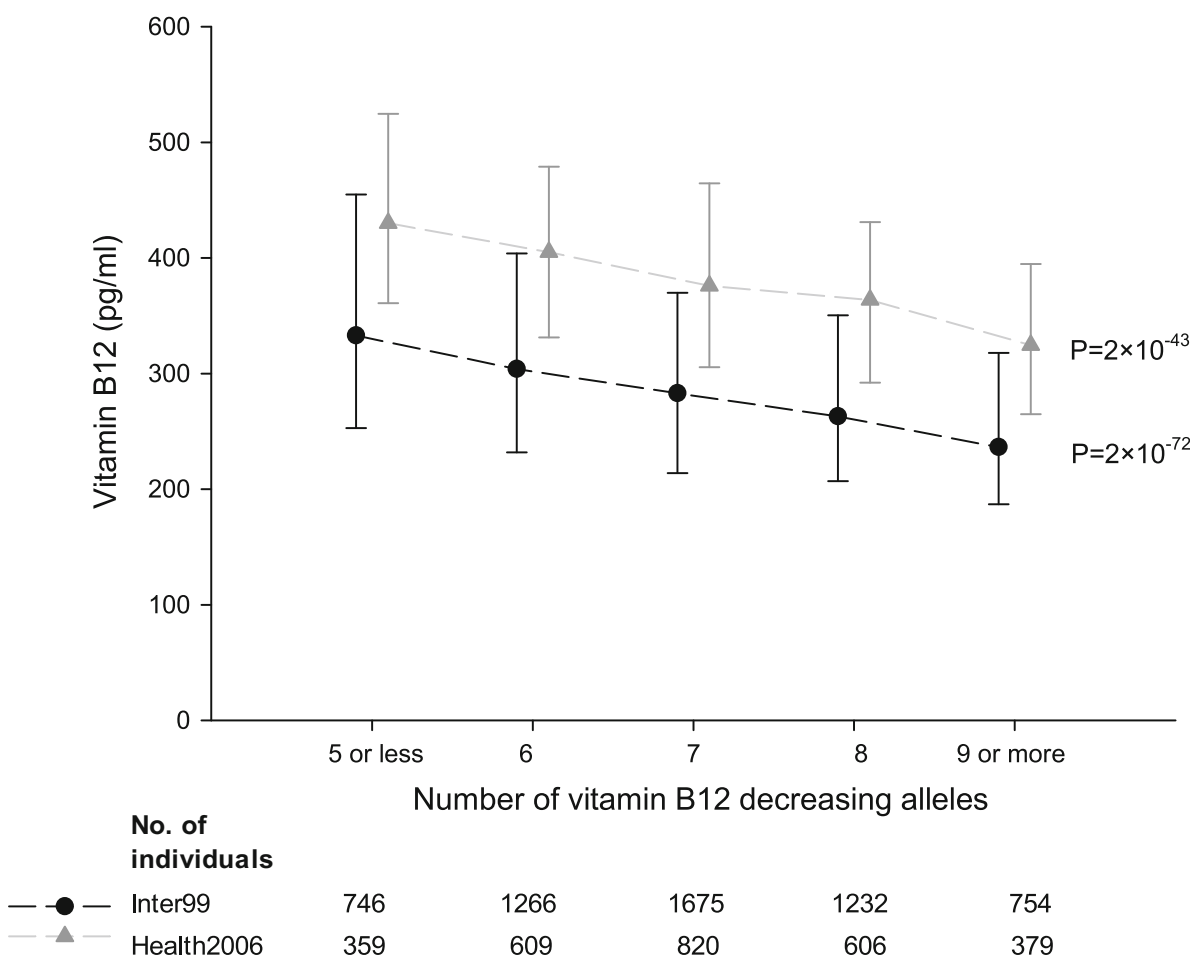

Table 2 Association between the B12 GRS and BMI

\begin{tabular}{llcc}
\hline & $\mathrm{n}$ & Weight $(\%)$ & Per allele effect, $\mathrm{kg} / \mathrm{m}^{2}(95 \% \mathrm{CI})$ \\
\hline B12 GRS & & & \\
Inter99 & 6076 & 68.5 & $-0.03(-0.11 ; 0.06)$ \\
Health2006 & 2854 & 31.5 & $0.04(-0.08 ; 0.17)$ \\
Fixed-effect model & & & $0.00(-0.07 ; 0.06)$ \\
Heterogeneity: $\mathrm{I}^{2}=0.0 \%, P=0.35$ & & & \\
\hline
\end{tabular}

Per allele effects are from linear regression analyses of the B12 GRS on BMI adjusted for age and sex

Fig. 3 Association between serum vitamin B12 levels and BMI. Squares indicate effect estimates from Inter99 and Health2006, sized according to the weight of each study in the meta-analyses. Diamonds indicate effect estimates from fixed-effects meta-analysis and error bars indicate $95 \% \mathrm{CI}$
Observational (a 20\% decrease in serum vitamin B12)

\begin{tabular}{|c|c|c|c|}
\hline Cohort & $\mathbf{n}$ & Weight, \% & Effect, $\mathrm{kg} / \mathrm{m}^{2}(95 \% \mathrm{Cl})$ \\
\hline Inter99 & 5969 & 67.5 & $0.08(0.03 ; 0.13)$ \\
\hline Health2006 & 3336 & 32.5 & $0.14(0.05 ; 0.23)$ \\
\hline \multicolumn{3}{|c|}{ Fixed-effect model } & $0.09(0.05 ; 0.13)$ \\
\hline
\end{tabular}

Genetic (a genetically induced $20 \%$ decrease in serum vitamin B12)

$\begin{array}{llll}\text { Cohort } & \mathbf{n} & \text { Weight, \% } & \text { Effect, } \mathbf{~ k g} / \mathbf{m}^{2}(\mathbf{9 5} \% \mathbf{C l} \\ \text { Inter99 } & 5670 & 76.0 & -0.09(-0.30 ; 0.13) \\ \text { Health2006 } & 2770 & 24.0 & 0.15(-0.23 ; 0.53) \\ \text { Fixed-effect model } & & -0.03(-0.22 ; 0.16) \\ \text { Heterogeneity, } P=12.5 \%, P=0.29 & & \end{array}$

Heterogeneity, $R=12.5 \%, P=0.29$

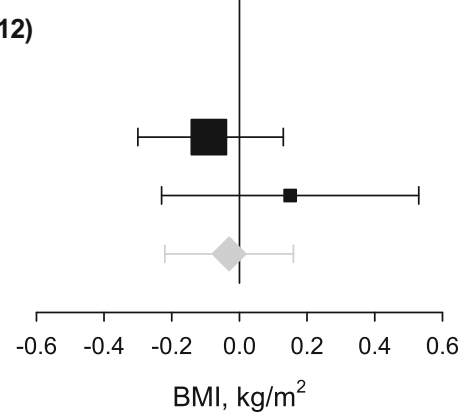


(Supplemental Table 3). The serum vitamin B12 decreasing FUT2 rs602662 G-allele associated with increased BMI when combining Inter99 and Health2006 in a fixed-effects meta-analysis $\left(0.15(95 \%\right.$ CI $\left.0.01 ; 0.32) \mathrm{kg} / \mathrm{m}^{2}, P=0.03\right)$, and the FUT2 genotype explained 0.05 and $0.02 \%$ of the phenotypic variance in BMI in Inter99 and Health2006, respectively.

\section{Validation analyses in two German cohorts}

Individuals in the German cohorts were middle-aged with slightly higher BMI than in Inter99 and Health2006 with a median BMI of 26.8 and $27.5 \mathrm{~kg} / \mathrm{m}^{2}$ for SHIP-0 and SHIPTREND, respectively (Supplemental Table 5). Serum vitamin B12 levels were not significantly associated with BMI (data not shown), but the serum vitamin B12 decreasing FUT2 rs602662 G-allele associated with increased BMI when combining SHIP-0 and SHIP-TREND in a fixed-effects meta-analysis [0.27 (95\% CI 0.09; 0.44) $\left.\mathrm{kg} / \mathrm{m}^{2}, \quad P=0.003\right]$. The FUT2 rs602662 genotype explained $0.12 \%$ of the phenotypic variance of BMI in both SHIP-0 and SHIP-TREND. When combining the Danish and the German cohorts, the FUT2 rs602662 showed a combined per G-allele effect of 0.19 (95\% CI 0.08 ; 0.30) $\mathrm{kg} / \mathrm{m}^{2}, P=4 \times 10^{-4}$ on BMI (Fig. 4). This association was supported by the European BMI data from the GIANT consortium where the FUT2 rs602662 G-allele $(\mathrm{n}=233,871)$ associated nominally with increased BMI $(\mathrm{P}=0.01)$.

\section{Sensitivity analyses}

Results from instrumental variable analysis where each of the eight SNPs were used as separate instruments (equivalent to a GRS weighted based on the SNP-serum vitamin B12 association) were similar to results from instrumental variable analysis based on the simple allele-counting B12 GRS (Supplemental Table 6; Fig. 3). Causal estimates from MR-Egger regression were largely similar to twostage least-squares regression estimates although the estimates from MR-Egger regression were underpowered (Supplemental Table 5; Supplemental Fig. 1). This was the case both when including and excluding FUT2 rs602662 and FUT6 rs778805. The intercept from MR-Egger regression did not differ from zero, neither when excluding FUT2 rs602662 and FUT6 rs778805, nor when including these two SNPs (Supplemental Table 6) suggesting that directional pleiotropy was not present. In support, Funnel plots were also largely symmetrical (Supplemental Fig. 1). No interaction was observed between the B12 GRS and BMI associated SNPs (Supplemental Table 7).

\section{Discussion}

We found an observational association between decreased serum vitamin B12 levels and elevated BMI in two Danish cohorts. This led to the hypothesis that low serum vitamin B12 levels are causally involved in body mass regulation, and we investigated this by applying a Mendelian randomization design, including a formal instrumental variable analysis using a B12 GRS to model an unbiased genetically induced decrease of serum vitamin B12. However, our results provided no clear evidence for a causal role of low serum vitamin B12 levels in obesity.

The Mendelian randomization design applied in this study may be likened to a randomized clinical trial, where individuals are randomized by nature to carry genetic variants associating with low or high circulating levels of vitamin B12. The purpose of the randomization process in a clinical trial is to obtain an even distribution of potential confounders across the treatment arms, and likewise the Mendelian randomization design should result in an equal distribution of known and unknown confounders across genotypes. Additionally, reverse causality (here that higher BMI causes lower serum vitamin B12 levels) is
Fig. 4 Association between FUT2 rs602662 and BMI. Squares indicate effect estimates from Inter99 and Health2006, sized according to the weight of each study in the meta-analyses. The diamonds indicates the effect estimate from fixed-effects meta-analysis and error bars indicate $95 \% \mathrm{CI}$

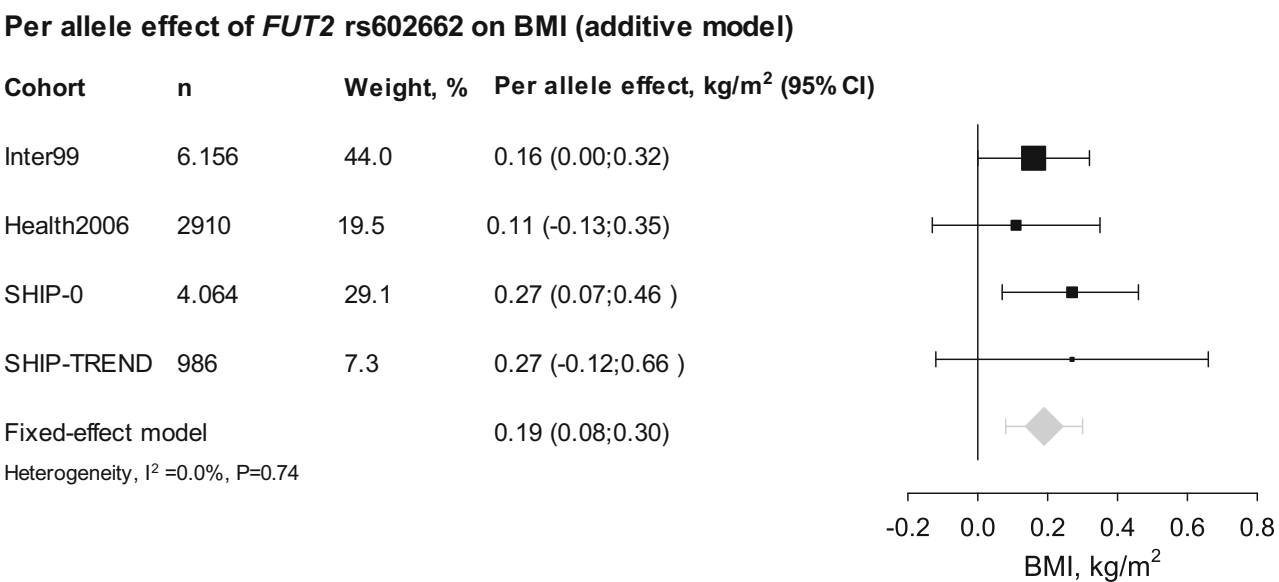

Per allele effect of FUT2 rs602662 on BMI (additive model)

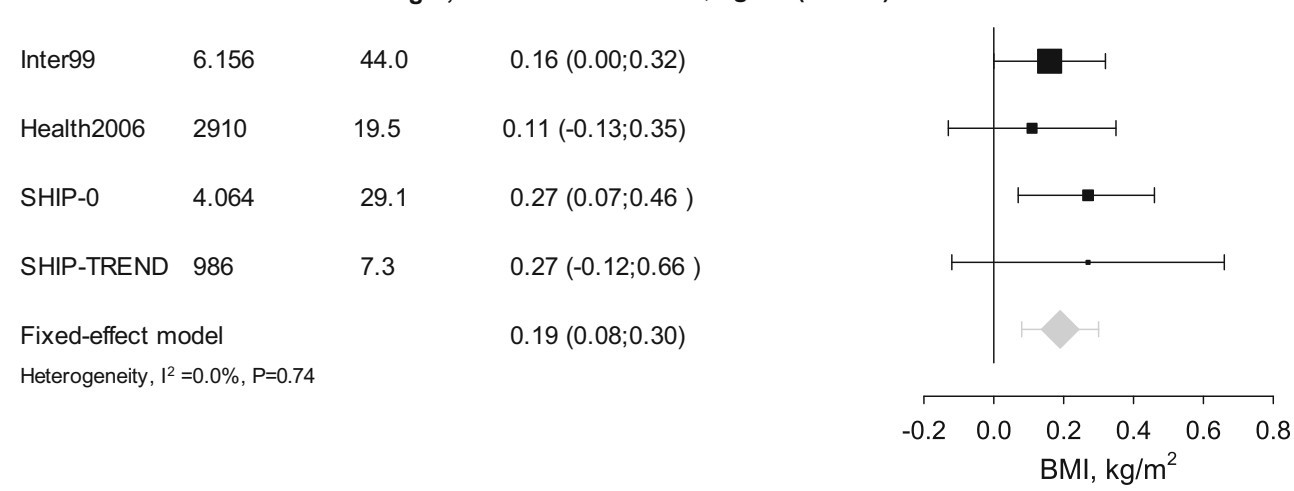


circumvented since genotypes are non-modifiable. Hence, in the setting of a Mendelian randomization study, genotypes or a GRS are used as an unconfounded instrument for the risk factor under investigation and allows a largely unbiased evaluation of the causal role of the risk factor. To obtain a proper instrument for serum vitamin B12 levels, we constructed a GRS consisting of eight vitamin B12 decreasing SNPs in genes encoding proteins from the pathway responsible for the absorption, processing, and coenzymatic activity of vitamin B12 [16]. This B12 GRS associated strongly with serum vitamin B12 and accordingly explained $\sim 6 \%$ of the phenotypic variance in serum vitamin B12 levels. For comparison, 97 BMI associated SNPs from the GIANT consortium cumulatively explain only $2.7 \%$ of the phenotypic variance in BMI [2], which among many reasons may be due to the unspecific representation of numerous different pathways where the variants are not directly linked functionally. The fact that the B12 GRS associated strongly and specifically with serum vitamin B12 levels and the fact that the eight SNPs included in the B12 GRS all have well-characterized roles in vitamin B12 functioning, are coding and thus potentially the true functional SNP, qualifies the B12 GRS as a useful instrument for evaluating causality. If serum vitamin B12 levels are causally linked to BMI, the B12 GRS should be related to BMI to the extent predicted by its influence on serum vitamin B12 levels. Although we did not have sufficient statistical power to completely rule out causality, our instrumental variable analyses provided no evidence that low serum vitamin B12 levels cause increased BMI.

A crucial assumption underlying the Mendelian randomization design is that the genotype is associated with the outcome only via the risk factor under investigation [17]. However, genetic variants may have pleiotropic effects and thus influence the outcome through other pathways than the one under investigation. To circumvent issues of pleiotropy, we therefore additionally applied the MR-Egger approach. Inclusion of FUT2 rs602662 and FUT6 rs778805 as instruments for genetically induced decreased serum vitamin B12 levels did not support introduction of directional pleiotropy, but the statistical power seemed to decrease markedly when applying the MR-Egger method. Nevetheless, FUT2 rs602662 which showed the strongest individual SNP effect on serum vitamin $\mathrm{B} 12$, is known to have pleiotropic effects. Thus, our finding that the G-allele of FUT2 rs602662 was associated with decreased levels of serum vitamin B12 as well as increased BMI, does not necessarily support a causal role of decreased vitamin B12 levels in obesity. This finding may be explained by the strong linkage disequilibrium $\left(\mathrm{r}^{2}=0.81, \mathrm{D}^{\prime}=1.0\right)$ between FUT2 $\mathrm{rs} 602662$ and FUT2 rs601338 which encodes a nonsense mutation (W143X) causing a lack of presentation of blood group antigens $\mathrm{A}, \mathrm{B}$ and $\mathrm{H}$ on mucosal surfaces and in secretions. This SNP could potentially modulate the composition and function of the gut microbiota, a hypothesis that is supported by various studies. Homozygous carriers of the stop codon (FUT2 rs601338 AA carriers corresponding to FUT2 rs602662 AA carriers) are nonsecretors and they have been shown to be resistant to infections with Helicobacter pylori $[27,28]$ and Norovirus, the major cause of viral acute gastroenteritis worldwide [29-32]. Infection with $H$. pylori may cause chronic gastritis [33] leading to decreased secretion of intrinsic factor and therefore impaired absorption of vitamin B12. Intriguingly, the resistance to gastrointestinal infections suggests that lack of blood group antigens on the intestinal mucosa not only inhibits the infectious potential of pathogenic gastrointestinal microbes, but also may inhibit the habitual interaction with commensal microbes in the gastrointestinal tract. This is supported by studies showing that secretor status influences the composition of the gut microbiota [34-36]. Also, several studies have reported a link between gut microbes and human obesity [37-39] and it has been demonstrated that germ-free mice are leaner than conventionally raised mice and that conventionalization of germfree mice with microbiota from conventionally raised mice results in an increased body fat content [37]. Therefore, the slightly lower BMI observed among AA carriers of FUT2 rs602662 (accompanied by higher serum vitamin B12 levels) in our study may be explained by the lack of expression of blood group antigens in the gastrointestinal tract and therefore impaired cross-talk with commensal microbes. In support of this interpretation, results from GIANT showed that the FUT2 rs601338 A-allele (coding for the stop codon) associated nominally with decreased BMI $(P=0.007)$. However, mechanistic studies in rodents and statistically powered studies of humans involving characterization of host genotype, microbiota composition, and BMI, must be conducted to fully address this hypothesis.

In conclusion, we did not find support for a causal role of decreased serum vitamin B12 levels in obesity. However, our study gives rise to the hypothesis that the FUT2 locus explains a part of the observational association between serum vitamin B12 and BMI through its regulation of the cross-talk between gut microbes and the human host. Furthermore, the B12 GRS constructed in this study may be used a valid instrument in future Mendelian randomization studies evaluating of the causal role of serum vitamin B12 levels in other disorders.

Acknowledgements We are indebted to the staff and participants of the Inter99, Health2006, SHIP-0 and SHIP-TREND studies for their important contributions. KHA and CHS were supported by grants from the Danish Council for Independent Research. 


\section{Compliance with ethical standards}

Conflict of interest The authors declare that they have no conflict of interest.

Open Access This article is distributed under the terms of the Creative Commons Attribution 4.0 International License (http://crea tivecommons.org/licenses/by/4.0/), which permits unrestricted use, distribution, and reproduction in any medium, provided you give appropriate credit to the original author(s) and the source, provide a link to the Creative Commons license, and indicate if changes were made.

\section{References}

1. Elks CE, den Hoed M, Zhao JH, Sharp SJ, Wareham NJ, Loos RJF, et al. Variability in the heritability of body mass index: a systematic review and meta-regression. Front Endocrinol (Lausanne). 2012;3:1-16. http://journal.frontiersin.org/article/10. 3389/fendo.2012.00029/abstract.

2. Locke AE, Kahali B, Berndt SI, Justice AE, Pers TH, Day FR, et al. Genetic studies of body mass index yield new insights for obesity biology. Nature. 2015;518:197-206. http://www.nature. com/doifinder/10.1038/nature14177.

3. Shungin D, Winkler TW, Croteau-Chonka DC, Ferreira T, Locke $\mathrm{AE}$, Mägi R, et al. New genetic loci link adipose and insulin biology to body fat distribution. Nature. 2015;518:187-96. http:// www.nature.com/doifinder/10.1038/nature14132.

4. Roth JR, Lawrence JG, Bobik TA. Cobalamin (coenzyme B12): synthesis and biological significance. Annu Rev Microbiol. 1996;50:137-81.

5. Finer S, Saravanan P, Hitman G, Yajnik C. The role of the onecarbon cycle in the developmental origins of type 2 diabetes and obesity. Diabet Med. 2014;31:263-72.

6. Kaya C, Cengiz SD, Satıroğlu H. Obesity and insulin resistance associated with lower plasma vitamin B12 in PCOS. Reprod Biomed Online. 2009;19:721-6. http://www.sciencedirect.com/ science/article/pii/S1472648309000200.

7. Pinhas-hamiel O, Doron-panush N, Reichman B, Nitzan-kaluski D, Shalitin S, Geva-lerner L. Obese children and adolescents: a risk group for low vitamin B12 concentration. Arch Pediatr Adolesc Med. 2006;160:933-6.

8. Knight BA, Shields BM, Brook A, Hill A, Bhat DS, Hattersley AT, et al. Lower circulating B12 is associated with higher obesity and insulin resistance during pregnancy in a non-diabetic white British population. PLoS One. 2015;10:e0135268. http://dx.plos. org/10.1371/journal.pone.0135268.

9. Rafnsson SB, Saravanan P, Bhopal RS, Yajnik CS. Is a low blood level of vitamin B12 a cardiovascular and diabetes risk factor? A systematic review of cohort studies. Eur J Nutr. 2011;50:97-106.

10. Krishnaveni GV, Hill JC, Veena SR, Bhat DS, Wills AK, Karat CLS, et al. Low plasma vitamin B12 in pregnancy is associated with gestational "diabesity" and later diabetes. Diabetologia. 2009;52:2350-8. http://link.springer.com/10.1007/s00125-0091499-0.

11. Nilsson SE, Read S, Berg S, Johansson B. Heritabilities for fifteen routine biochemical values: findings in 215 Swedish twin pairs 82 years of age or older. Scand J Clin Lab Investig. 2009; epub:1-8. 19343610.

12. Hazra A, Kraft P, Selhub J, Giovannucci EL, Thomas G, Hoover $\mathrm{RN}$, et al. Common variants of FUT2 are associated with plasma vitamin B12 levels. Nat Genet. 2008;40:1160-2. http://www.nat ure.com/ng/journal/v40/n10/full/ng.210.html\#B10.
13. Hazra A, Kraft P, Lazarus R, Chen C, Chanock SJ, Jacques P, et al. Genome-wide significant predictors of metabolites in the one-carbon metabolism pathway. Hum Mol Genet. 2009;18: 4677-87. http://www.pubmedcentral.nih.gov/articlerender.fcgi?artid= 2773275\&tool $=$ pmcentrez\&rendertype $=$ abstract.

14. Lin X, Lu D, Gao Y, Tao S, Yang X, Feng J, et al. Genome-wide association study identifies novel loci associated with serum level of vitamin B12 in Chinese men. Hum Mol Genet. 2012;21:2610-7. http://www.hmg.oxfordjournals.org/cgi/doi/10. 1093/hmg/dds062.

15. Tanaka T, Scheet P, Giusti B, Bandinelli S, Piras MG, Usala G, et al. Genome-wide association study of vitamin B6, vitamin B12, folate, and homocysteine blood concentrations. Am J Hum Genet. 2009;84:477-82. http://linkinghub.elsevier.com/retrieve/ pii/S0002929709000974.

16. Grarup N, Sulem P, Sandholt CH, Thorleifsson G, Ahluwalia TS, Steinthorsdottir V, et al. Genetic architecture of vitamin B12 and folate levels uncovered applying deeply sequenced large datasets. PLoS Genet. 2013;9:e1003530. http://www.pubmedcentral.nih. gov/articlerender.fcgi?artid=3674994\&tool=pmcentrez\&render type $=$ abstract.

17. Davey Smith G, Hemani G. Mendelian randomization: genetic anchors for causal inference in epidemiological studies. Hum Mol Genet. 2014;23:R89-98. http://www.hmg.oxfordjournals.org/cgi/ doi/10.1093/hmg/ddu328.

18. Thuesen BH, Husemoen LLN, Ovesen L, Jørgensen T, Fenger M, Linneberg A. Lifestyle and genetic determinants of folate and vitamin B12 levels in a general adult population. Br J Nutr. 2010;103:1195-204.

19. Jorgensen T, Borch-Johnsen K, Thomsen TF, Ibsen H, Glumer C, Pisinger C. A randomized non-pharmacological intervention study for prevention of ischaemic heart disease: baseline results Inter99 (1). Eur J Cardiovasc Prev Rehabil. 2003;10:377-86. http://cpr.sagepub.com/lookup/doi/10.1097/01.hjr.0000096541.30 533.82 .

20. Thuesen BH, Cerqueira C, Aadahl M, Ebstrup JF, Toft U, Thyssen JP, et al. Cohort profile: the Health2006 cohort, research centre for prevention and health. Int J Epidemiol. 2014;43: $568-75$.

21. Völzke H, Alte D, Schmidt O, Radke D, Lorbeer R, Friedrich N, et al. Cohort profile: the study of health in Pomerania. Int J Epidemiol. 2011;40:294-307.

22. Toft U, Kristoffersen L, Ladelund S, Bysted A, Jakobsen J, Lau $\mathrm{C}$, et al. Relative validity of a food frequency questionnaire used in the Inter99 study. Eur J Clin Nutr 2008;62:1038-46. http:// www.nature.com/doifinder/10.1038/sj.ejen.1602815.

23. von Huth Smith L, Borch-Johnsen K, Jørgensen T. Commuting physical activity is favourably associated with biological risk factors for cardiovascular disease. Eur J Epidemiol. 2007;22: 771-9. http://www.ncbi.nlm.nih.gov/pubmed/17846902.

24. Pisinger C, Glümer C, Toft U, von Huth Smith L, Aadahl M, Borch-Johnsen $\mathrm{K}$, et al. High risk strategy in smoking cessation is feasible on a population-based level. The Inter99 study. Prev Med (Baltim). 2008;46:579-84. http://www.sciencedirect.com/sci ence/article/pii/S0091743508001138.

25. The 1000 Genomes Project Consortium. An integrated map of genetic variation. Nature. 2012;491:56-65.

26. Bowden J, Smith GD, Burgess S. Mendelian randomization with invalid instruments: effect estimation and bias detection through Egger regression. Int J Epidemiol. 2015;44:512-25. http://www. ncbi.nlm.nih.gov/pubmed/26050253.

27. Azevedo M, Eriksson S, Mendes N, Serpa J, Figueiredo C, Resende $\mathrm{LP}$, et al. Infection by Helicobacter pylori expressing the BabA adhesin is influenced by the secretor phenotype. J Pathol 2008;215:308-16. http://www.ncbi.nlm.nih.gov/pubmed/18498114. 
28. Ikehara Y, Nishihara S, Yasutomi H, Kitamura T, Matsuo K, Shimizu N, et al. Polymorphisms of two fucosyltransferase genes (Lewis and Secretor genes) involving type I Lewis antigens are associated with the presence of anti-Helicobacter pylori IgG antibody. Cancer Epidemiol Biomarkers Prev. 2001;10:971-7. http://www.ncbi.nlm.nih.gov/pubmed/11535550.

29. Thorven M, Grahn A, Hedlund K-O, Johansson H, Wahlfrid C, Larson $\mathrm{G}$, et al. A homozygous nonsense mutation $(428 \mathrm{G} \rightarrow \mathrm{A})$ in the human secretor (FUT2) gene provides resistance to symptomatic norovirus (GGII) infections. J Virol. 2005;79:15351-5. http://www.pubmedcentral.nih.gov/articlerender.fcgi?artid=1315 998\&tool=pmcentrez\&rendertype $=$ abstract.

30. Lindesmith L, Moe C, Marionneau SEM, Ruvoen NA, Jiang XI, Lindblad LA, et al. Human susceptibility and resistance to Norwalk virus infection. Nat Med. 2003;9:548-53. http://www.ncbi. nlm.nih.gov/entrez/query.fcgi? $\mathrm{cmd}=$ Retrieve $\& \mathrm{db}=$ PubMed\&dopt $=$ Citation\&list_uids $=12692541$.

31. Lindesmith LC, Donaldson EF, LoBue AD, Cannon JL, Zheng D-P, Vinje J, et al. Mechanisms of GII.4 norovirus persistence in human populations. PLoS Med. 2008;5:e31. http://www.plosme dicine.org/article/info:doi/10.1371/journal.pmed.0050031.

32. Larsson MM, Rydell GEP, Grahn A, Rodriguez-Diaz J, Akerlind $\mathrm{B}$, Hutson AM, et al. Antibody prevalence and titer to norovirus (genogroup II) correlate with secretor (FUT2) but not with ABO phenotype or Lewis (FUT3) genotype. J Infect Dis. 2006;194:1422-7.

33. Hunt RH, Camilleri M, Crowe SE, El-Omar EM, Fox JG, Kuipers $\mathrm{EJ}$, et al. The stomach in health and disease. Gut.
2015;64:1650-68. http://gut.bmj.com/lookup/doi/10.1136/gutjnl2014-307595.

34. Rausch P, Rehman A, Künzel S, Häsler R, Ott SJ, Schreiber S, et al. Colonic mucosa-associated microbiota is influenced by an interaction of Crohn disease and FUT2 (Secretor) genotype. Proc Natl Acad Sci USA. 2011;108:19030-5. http://www.pub medcentral.nih.gov/articlerender.fcgi?artid=3223430\&tool=pmcen trez\&rendertype $=$ abstract.

35. Wacklin P, Mäkivuokko H, Alakulppi N, Nikkilä J, Tenkanen H, Räbinä J, et al. Secretor genotype (FUT2 gene) is strongly associated with the composition of bifidobacteria in the human intestine. PLoS One 2011;6:e20113. http://dx.plos.org/10.1371/ journal.pone.0020113.

36. Lewis ZT, Totten SM, Smilowitz JT, Popovic M, Parker E, Lemay DG, et al. Maternal fucosyltransferase 2 status affects the gut bifidobacterial communities of breastfed infants. Microbiome. 2015;3:13. http://www.microbiomejournal.com/content/3/1/13.

37. Bäckhed F, Ding H, Wang T, Hooper LV, Koh GY, Nagy A, et al. The gut microbiota as an environmental factor that regulates fat storage. Proc Natl Acad Sci USA. 2004;101:15718-23.

38. Turnbaugh PJ, Hamady M, Yatsunenko T, Cantarel BL, Duncan A, Ley RE, et al. A core gut microbiome in obese and lean twins. Nature. 2009;457:480-4. http://www.nature.com/doifinder/10. 1038 /nature 07540 .

39. Le Chatelier E, Nielsen T, Qin J, Prifti E, Hildebrand F, Falony $\mathrm{G}$, et al. Richness of human gut microbiome correlates with metabolic markers. Nature. 2013;500:541-6. http://www.nature. com/doifinder/10.1038/nature12506. 